\title{
Mycobacterium avium Pulmonary Disease with Non-Specific Chest Imaging Findings
}

\section{Jin-Fu Xu' ${ }^{1}$, Hui-Ping $\mathrm{Li}^{1 *}$ and Jiro Fujita ${ }^{2}$}

${ }^{1}$ Department of Respiratory Medicine, Shanghai Pulmonary Hospital, Tongji University School of Medicine, Shanghai, 200433, China

${ }^{2}$ Department of Infectious, Respiratory, and Digestive Medicine, University of the Ryukyus, Okinawa, 903-0215, Japan

\begin{abstract}
A 20-year-old female without immune-compromise was referred to our clinic complaining of cough with hemoptysis. Chest radiology showed multiple small lung nodules at the upper lobe of bilateral lung. After received regular anti-tuberculosis therapy and experiential anti-fungal therapy, the lesions in lung get worse. The biopsy was pursued and the diagnosis was made through lung tissue-based mycobacterial culture. It is difficult to differentiate Mycobacterium avium pulmonary disease with non-specific imaging findings from lung fungal infection and pulmonary tuberculosis.
\end{abstract}

Keywords: Mycobacterium avium; Non-tuberculous mycobacterial pulmonary disease; Herxheimer-like reaction; Non-immunocompromise; Lung biopsy

\section{Introduction}

Although the epidemiology of pulmonary Tuberculosis (TB) is well described, the prevalence and epidemiology of Non-Tuberculous Mycobacterial (NTM) lung disease is largely unknown. The incidence of NTM is increasing in the non-HIV population [1-3]. The Official Statement of the American Thoracic Society (ATS) and the Infectious Diseases Society of America (IDSA) on NTM [4] described that the minimum evaluation of a patient suspected of NTM lung disease should include the following: (1) chest radiograph or, in the absence of cavitation, chest High-Resolution Computed Tomography (HRCT) scan; (2) three or more sputum specimens for Acid-Fast Bacilli (AFB) analysis; and (3) exclusion of other disorders, such as TB. Confirmed diagnosis should include further specific characters such as clinical, radiologic, and microbiologic criteria. Here we report a case of pulmonary NTM disease proved by lung biopsy with non-specific imaging findings in a non-immunocompromised patient.

\section{Case Report}

A 20-year-old female student was admitted to hospital for cough with hemoptysis one month. One month ago, the patient developed intermittent cough with no obvious incentive. The cough was paroxysmal intensified at day time and accompanied with hemoptysis. It was bright red blood and blood clots could be seen sometimes. It was approximately $200 \mathrm{ml}$ per day, without stomach contents, no fever, no chest and abdominal pain, no diarrhea, no chest tightness, no shortness of breath and no heart palpitations.

She was diagnosed with community acquired pneumonia but she was still bleeding after repeated antibiotics treatment. A chest CT scanning (Figure 1A) showed multiple small lung nodules in lung, mainly at the upper area of bilateral sides, with minor bronchiectasis in the middle lobe of right lung. The medical history showed that she had no any accompanied disease. No immunocompromised state existed. No superficial lymph node enlargement; no fever, coarse breath sounds in bilateral lungs, no wet and dry rales. Regular experimental tests were shown at Table 1. The diagnosis was supposed to be pulmonary tuberculosis. She began to receive experiential short-course chemotherapy strategy for tuberculosis, which includes regular dosages of isoniazid, rifampin, streptomycin and pyrazinamide. On the second day, the hemoptysis decreased and seldom occurred thereafter.

However, after about two months' therapy, she still had intermittent
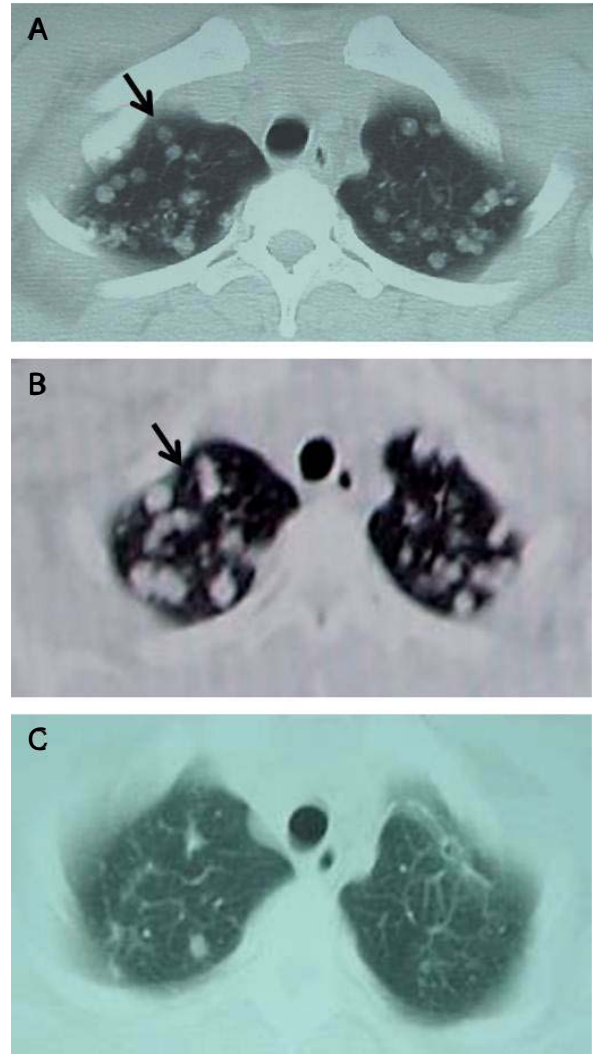

Figure 1: $A$ to $C$ showed the changes of main lesions of the lung by chest $C T$ scan. A, CT showed small nodules in the upper lobes of lung before treatment; $\mathrm{B}$, nodules enlarged after anti-tuberculosis therapy; C, nodules were dissipation after anti-M. avium therapy.

${ }^{*}$ Corresponding author: Hui-Ping Li, 507 Zhengmin Road, Department of Respiratory Medicine, Shanghai Pulmonary Hospital, Tongii University School of Medicine, Shanghai, 200433, China, Tel: +86 21 65115006; Fax: +86 21 55663289; E-mail: lihuiping1958@yahoo.com.cn

Received March 30, 2012; Accepted May 11, 2012; Published May 13, 2012

Citation: Xu JF, Li HP, Fujita J (2012) Mycobacterium avium Pulmonary Disease with Non-Specific Chest Imaging Findings. J Pulmon Resp Med 2:123. doi:10.4172/2161-105X.1000123

Copyright: (c) $2012 \mathrm{Xu} \mathrm{JF}$, et al. This is an open-access article distributed under the terms of the Creative Commons Attribution License, which permits unrestricted use, distribution, and reproduction in any medium, provided the original author and source are credited. 


\begin{tabular}{|l|l|}
\hline Item & Results \\
\hline Peripheral blood & Normal \\
\hline Blood Routine & $89 \mathrm{~mm} / \mathrm{h}$ \\
\hline ESR & POS \\
\hline Anti-tuberculosis IgG & NEG \\
\hline Cryptococcal latex agglutination test & NEG \\
\hline Anti-HIV antibody & 1.87 \\
\hline CD4+ T cell/CD8+ T cell & POS \\
\hline Galactomannan test & POS \\
\hline Beta-D-glucan test & \\
\hline Sputum culture & NEG \\
\hline Bacteria & NEG \\
\hline Fungi & NEG \\
\hline Tuberculosis & NEG \\
\hline Sputum swear for acid fast stain & $10 \times 10 \mathrm{~mm}$ \\
\hline Tuberculin purified protein derivative skin test & \\
\hline
\end{tabular}

POS, positive; NEG, negative

Table 1: Initial laboratory finding of the patient.

cough with occasional hemoptysis. She received the second chest CT scan and it showed that the nodules were not dissipated but increased, with a film-like plaques, nodules, irregular shape, uneven density within the boundary not very clear (Figure $1 \mathrm{~B}$ ). She received more tests for the diagnosis. Chest X-ray showed multiple small lung nodules in lung, with density uneven. For differential diagnose of fungal infection, blood fungal antigens were detected. The Galactomannan test (GM test) and beta-D-glucan ( $\mathrm{G}$ test) were performed as manufactory suggested. The GM tests in blood were positive twice and G test in bronchoalveolar lavage fluid was positive also. Based on the results of antigen detections, the diagnosis of pulmonary fungal disease especially aspergillus can not exclude according to the guideline of ATS/IDSA [5]. She received experiential anti-aspergillus therapy due to probable diagnosis of pulmonary aspergillosis without stop the anti-tuberculosis drugs.

Two weeks later, she got a recurrence of hemoptysis. To identify the diagnosis, the patient received Video-Assisted Thoracoscopic Surgery (VATS) for lung biopsy. Her lung pathology showed pathological granulomatous lesions with non-specific central necrosis in HE stained slides (Figure 2A). The PAS staining and methenamine silver staining of biopsied tissue were negative. Pathologist found the acid-fast positive bacilli in the tissue using acid-fast stain (Figure 2B). But the lesions of patient still couldn't get better after the active anti-tuberculosis therapy. Finally what we suspected was proved by the tissue culture and identified by 16s RNA gene sequencing. The results showed it was $M$. avium. She received continuous anti-NTM treatment based on the drug susceptibility test. The duration was suggested to 18 months but she was followed up for only 9 months. Drugs include Rifapentine, Ethambutol, Azithromycin and Isoniazid. The lesions were dissipation finally (Figure 1C).

\section{Discussion}

This case illustrates several difficulties in the diagnosis of pulmonary NTM. Recent report showed that M. avium pulmonary disease is not rare, particularly among females [2]. In most patients, we could not make a right diagnosis based on sputum smear and culture, because sputum smear and culture usually were negative. Several methods such as serological antibody detection and Polymerase Chain Reaction (PCR) etc. were developed to improve the diagnosis possibility [68]. But it is still remain unsatisfactory in the early diagnosis of NTM. When the diagnosis can not be determined and the patient's situation begins to get worse, the lung biopsy is needed somehow. Early diagnosis is crucial for providing the patient right treatment and good prognosis.

Secondly, the positive result of acid-fast stain could not be regarded as mycobacterium tuberculosis infection only. Non-tuberculosis, $M$. leprae and Nocardia bacteria are also stain positive by acid-fast stain. As we know, granulomatous lung diseases are a special kind of proliferative lesions. The etiologies are various, in which mycobacterium, sarcoidosis and fungal infections are the common causes of them. If we combined pathological Hematoxylin-Eosin (H.E.) staining with specific staining such as acid-fast stain, PAS stain and methenamine silver staining, etc. for differentiating the diagnoses, the positive rates can be improved.

Meanwhile, it is important to send samples acquired from biopsy to bacterial culture and identify the type of bacterium. Sometimes it is difficult to identify the pathogen only by pathology and methods of special stains. In this case it is difficult to make the diagnosis of pulmonary NTM if we didn't get the result of bacterium culture and identification from her lung biopsy samples. NTM has a higher rate of drugs resistance. Results of bacteria-type identification and drug susceptibility are the guidance for treatment options. The effectiveness of anti-NTM treatment in patients with NTM was relatively slow [9].

Sometimes in the follow-up of the treatments of pulmonary
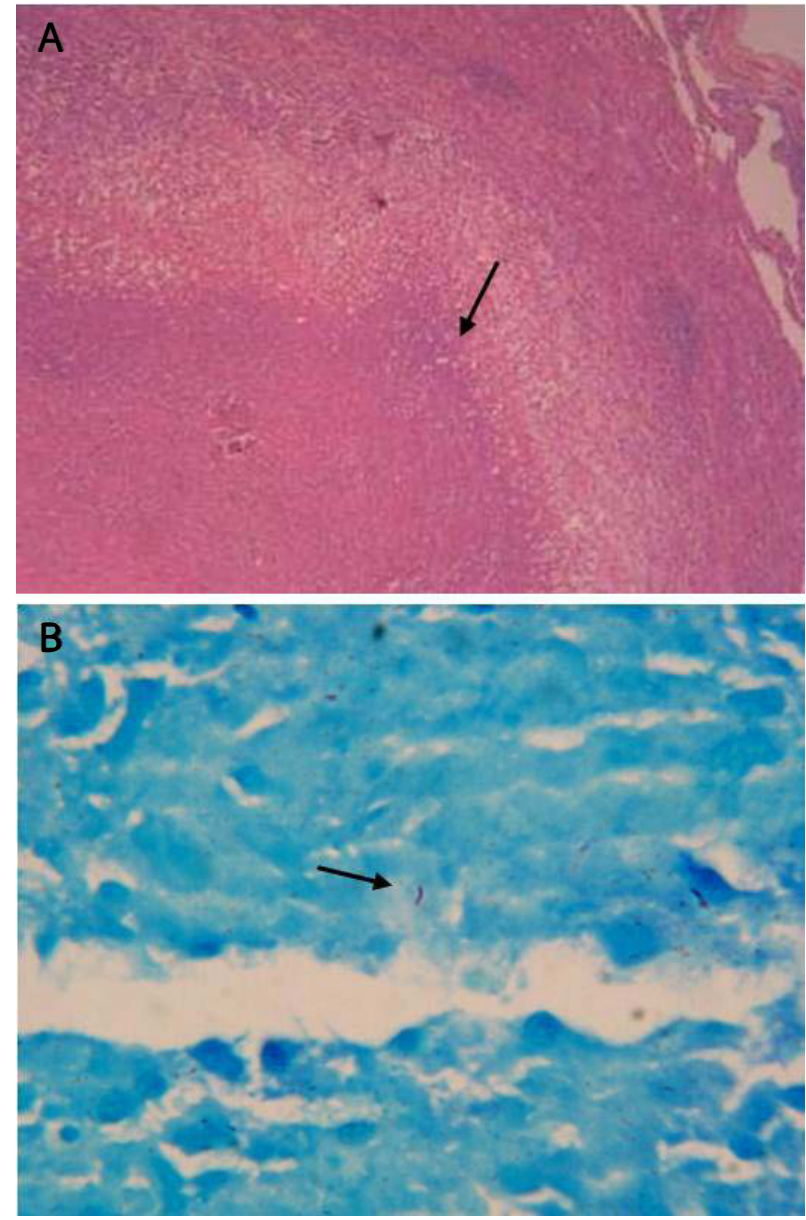

Figure 2: A, HE stain of lung biopsy sample showed the granulomatous lesions with non-specific central necrosis, H.E. stain, $\times 100 ; B$, acid-fast staining with lung biopsy sample, $\times 400$. Arrow showed acid-fast staining positive bacilli in the tissue. 
mycobacterial diseases, the lesions become larger than before. This phenomenon can be observed in various kinds of mycobacterial infections, which named paradoxical expansion, or Herxheimer-like reaction, due to increase lesions after the anti-mycobacterial therapy. In this case, we can not decide that it was the paradoxical expansion or not, because the treatment was not exactly the right one at that time. But by upholding anti-M. avium treatment, the lesions absorbed finally.

Fungal antigens tests have prospects in the diagnosis of fungal infections. Several guidelines suggest a good sensitivity and specificity of $\mathrm{G}$ test and GM test for diagnosing pulmonary fungal infection $[5,10]$. However, factors such as albumin or globulin injection, hemodialysis, perioperative period, specimens contact with gauze, allogeneic bone marrow transplantation and the use of semi-synthetic penicillin, etc. would cause G test or GM test prone to false positive [11]. In this case, the patient was tested positive in both G test and GM test, probably resulted from previously piperacillin use, provided a possible diagnosis of invasive pulmonary aspergillosis with clinical microbiology evidence. But the PAS staining and methenamine silver staining of biopsied tissue were negative. She was pathologically proved that it was not aspergillus infection but M. avium infection. Kobashi et al. [12] had reported 9 cases of Chronic Necrotizing Pulmonary Aspergillosis (CNPA) as a complication of $M$. avium pulmonary disease. The two diseases need to be differentiated carefully.

In short, we have described a case of lung biopsy proved $M$. avium pulmonary disease without specific imaging findings in nonimmunocompromised patient. NTM is not rare even in patients with normal immune states. If biopsy was pursued, tissue-based pathogen cultures are helpful to identify the pathogen, especially for the diagnosis of NTM with non-specific imaging findings.

\section{Acknowledgements}

The study was funded by Shanghai Subject Chief Scientist (No: 09411951500), National Science Foundation of China (No. 30971323 and No. 81170003), National 973 (NO.2010CB945600, 2010CB945601) and Shanghai Elite Young Physician Project (NO. XYQ2011006).

\section{References}

1. Fritscher LG, Marras TK, Bradi AC, Fritscher CC, Balter MS, et al. (2011) Nontuberculous mycobacterial infection as a cause of difficult-to-control asthma: a case control study. Chest 139: 23-27.

2. Marras TK, Chedore P, Ying AM, Jamieson F (2007) Isolation prevalence of pulmonary non-tuberculous mycobacteria in Ontario, 1997-2003. Thorax 62 : 661-666.

3. Winthrop KL, McNelley E, Kendall B, Marshall-Olson A, Morris C, et al. (2010) Pulmonary nontuberculous mycobacterial disease prevalence and clinical features; an emerging public health disease. Am J Respir Crit Care Med 182 977-982

4. Griffith DE, Aksamit T, Brown-Elliott BA, Catanzaro A, Daley C, et al. (2007) An official ATS/IDSA statement: diagnosis, treatment, and prevention of nontuberculous mycobacterial diseases. Am J Respir Crit Care Med 175: $367-$ 416 .

5. Walsh TJ, Anaissie EJ, Denning DW, Herbrecht R, Kontoyiannis DP, et al. (2008) Treatment of aspergillosis: clinical practice guidelines of the Infectious Diseases Society of America. Clin Infect Dis 46: 327-360.

6. Suzuki T, Tanaka M, Otani S, Matsuura S, Sakaguchi Y, et al. (2006) New rapid detection test with a combination of polymerase chain reaction and immunochromatographic assay for Mycobacterium tuberculosis complex. Diagn Microbiol Infect Dis 56: 275-280.

7. Olsen SR, Long R, Tyrrell G, Kunimoto D (2010) Induced sputum for the diagnosis of pulmonary tuberculosis: Is it useful in clinical practice? Can Respir J 17: e81-e84.

8. Steingart KR, Henry M, Laal S, Hopewell PC, Ramsay A, et al. (2010) Commercial serological antibody detection tests for the diagnosis of pulmonary tuberculosis: a systematic review. PLoS Med 4: e202.
9. Cassidy PM, Hedberg K, Saulson A McNelly E, Winthrop KL (2009) Nontuberculous mycobacterial disease prevalence and risk factors: a changing epidemiology. Clin Infect Dis 49: e124-e129.

10. Pappas PG, Kauffman CA, Andes D, Benjamin DK Jr, Calandra TF, et al. (2009) Clinical practice guidelines for the management of candidiasis: 2009 update by the Infectious Diseases Society of America. Clin Infect Dis 48: 503-535.

11. Khan ZU, Ahmad S, Theyyathel AM (2008) Detection of Aspergillus fumigatusspecific DNA, (1-3)-beta-D-glucan and galactomannan in serum and bronchoalveolar lavage specimens of experimentally infected rats. Mycoses 51: 129-135.

12. Kobashi Y, Fukuda M, Yoshida K, Miyashita N, Niki Y, et al. (2006) Chronic necrotizing pulmonary aspergillosis as a complication of pulmonary Mycobacterium avium complex disease. Respirology 11: 809-813. 